\title{
Correlación entre acciones de permanencia estudiantil y la resonancia de marca en una institución de educación superior
}

\author{
Correlation between student retention actions and brand resonance in a higher \\ education institution
}

\section{Correlação entre ações de retenção de estudantes e ressonância de marca em uma instituição de ensino superior}

\author{
Andrés David Gutiérrez Torres* | Laura Mercedes Moreno Hernández \\ Fundación Universitaria Konrad Lorenz, Bogotá D.C., Colombia
}

- Fecha de recepción: 2019-1I-12

- Fecha concepto de evaluación: 2019-11-13

- Fecha de aprobación: 2019-11-25 http://dx.doi.org/I0.22335/rlct.vI2il.1098
Para citar este artículo / To reference this article / Para citar este artigo: GutiérrezTorres, A. D., \& Moreno-Hernández, L. M. (2020). Correlación entre acciones de permanencia estudiantil y la resonancia de marca en una institución de educación superior. Revista Logos

Ciencia \& Tecnología, I2(I), 85-94. http://dx.doi.org// 0.22335/rlct.v I 2il. 1098

\begin{abstract}
RESUMEN
Se realizó una investigación para identificar la relación entre el valor de marca de acuerdo con el modelo de Keller, Brand Equity basado en el consumidor, y la intención del estudiante de permanecer en la institución donde adelanta sus estudios de pregrado. Se desarrolló un estudio descriptivo correlacional, utilizando un muestreo no probabilístico con 453 participantes que respondieron a una encuesta especialmente diseñada para el estudio, la cual incluyó las variables utilizadas por el Ministerio de Educación Nacional colombiano para medir acciones de permanencia, así como variables afines al modelo de resonancia de marca. Los resultados más relevantes sugieren una asociación positiva entre los procesos de internacionalización y la intención de permanencia en la institución; correlación entre la percepción de las acciones de permanencia y el valor de la marca en las instituciones de educación superior (IES). Asimismo, se evidencia fuerte conexión entre el modelo de resonancia de la marca Keller y el modelo de abandono institucional de Tinto que permite el fortalecimiento de la permanencia y la reducción de la deserción estudiantil.
\end{abstract}

Palabras clave: resonancia de marca, psicología del consumidor, permanencia estudiantil, educación superior 


\begin{abstract}
SUMMARY
A research relating Consumer Based Brand Equity and students' permanence intention in the educative institution, was performed. A descriptive correlational study was carried out, using a non-probabilistic sampling with 453 participants in total, which answered a survey that included the variables used by Colombian National Education Ministry (Ministerio de Educación Nacional de Colombia) to measure permanence actions as well as other variables related with the brand equity model, which were specifically developed for the main purposes of this study. The most important results suggest a positive association between internationalization processes and the permanence intention in the institution; a correlation between perception of the permanence actions and the brand equity in the Superior Education Institution (IES, because of their acronym in Spanish); in the same way, a strong connection between Keller's Brand resonance model and the Institutional Abandonment of Tinto's. This evidenced relationship would allow the strengthen of permanence and lowering of student's abandonment.
\end{abstract}

Keywords: Brand equity, consumer psychology, permanence college, higher education

\title{
SUMÁRIO
}

Uma investigação foi conduzida para identificar a relação entre o valor da marca, de acordo com o modelo de Keller, o Brand Equity do Consumidor e a intenção do aluno de permanecer na instituição onde estuda. Foi desenvolvido um estudo descritivo correlacional, utilizando uma amostra não probabilística com 453 participantes que responderam a uma pesquisa especialmente elaborada para o estudo, que incluiu as variáveis utilizadas pelo Ministério da Educação Nacional da Colômbia para medir ações de permanência, bem como variáveis relacionadas a modelo de ressonância de marca. Os resultados mais relevantes sugerem uma associação positiva entre os processos de internacionalização e a intenção de permanecer na instituição; correlação entre a percepção das ações de permanência e o valor da marca nas instituições de ensino superior (IES). Da mesma forma, existe uma forte conexão entre o modelo de ressonância da marca Keller e o modelo de abandono institucional da Tinto, que permite o fortalecimento da permanência e a redução do abandono escolar.

Palavras-chave: ressonância de marca, psicologia do consumidor, permanência de estudantes, ensino superior

Recientemente, las instituciones de educación superior (IES) en el mundo han tenido una constante preocupación por disminuir los índices de deserción estudiantil, teniendo en cuenta que el proceso de universalización y comercialización de la educación superior ha tenido el objetivo de masificarse para ampliar el acceso de toda la población. Aunque la educación sigue siendo un privilegio para quienes tienen los suficientes recursos económicos para cubrir los gastos e inversiones relacionados con el proceso de educación superior. Hacia finales del siglo $X X$, la educación comienza a ser un asunto más accesible e inclusivo de lo que había sido (Denegri, Cabezas, Novoa, Peralta \& Estrada, 20I3). Según cifras del Banco Mundial (2017), el acceso a la educación superior en América Latina se incrementó del $21 \%$ en el año 2000 al $40 \%$ en el 2010 y en general, el acceso a universidades privadas au- mentó al $50 \%$ en el 2013 ; este crecimiento ha provocado que las instituciones consideren nuevas estrategias a nivel organizacional, social y de calidad para hacerle frente al tema de la deserción estudiantil, no solo por la idea de la universalidad en la educación, sino también porque la educación, en términos económicos y comerciales, es un campo cada vez más competitivo y, en ese sentido, las universidades enfrentan retos importantes a la hora de buscar nuevos estudiantes, lograr la retención (permanencia) de los mismos y a largo plazo que sus egresados vuelvan a ellas. Por ello, son cada vez más las universidades que son conscientes de gestionar mejores relaciones entre los estudiantes y la percepción que estos tienen de la marca de la IES. 
A continuación, se presentan los modelos de permanencia estudiantil contemplados por instituciones de educación superior, sus características principales y su pertinencia para los hallazgos del presente estudio.

\section{Permanencia estudiantil}

Según el Ministerio de Educación Nacional (MEN) (2009), el término "permanencia" se refiere al éxito académico del estudiante y se representa en la culminación exitosa de los estudios emprendidos; en ese sentido, se manifiesta en una conducta que se mantiene en el tiempo y tiene que ver con la lealtad a la marca o el servicio, el sentido de pertenencia y la proyección. EI MEN considera los siguientes factores como aspectos cruciales para promover la permanencia estudiantil $y$, por ende, para la reducción de la deserción estudiantil en educación superior: acompañamiento académico y refuerzos que fortalecen los conocimientos con los cuales vienen los estudiantes, apoyos económicos (becas, subsidios, créditos), orientación vocacional, actitudes y cumplimiento efectivo de expectativas.

A su vez, Berger, Blanco y Lyon (20l2) plantean que la permanencia es el deseo y acción de los estudiantes por quedarse dentro del sistema de la educación superior con el fin de obtener su título académico; los autores proponen diferenciar entre permanencia y retención estudiantil, que se refiere a la cantidad de acciones que realiza la institución para que los estudiantes logren la permanencia, desde la admisión hasta su graduación. De igual forma, Hundrieser (2008) citado por Torres (2012), plantea que la permanencia estudiantil se define como la finalización exitosa de los objetivos académicos y tiene que ver con las características educativas, motivacionales y sociales del estudiante.

Fonseca y García (2016) proponen estudiar la deserción desde un enfoque organizacional, pues esto permite comprender que la decisión respecto a la permanencia no depende totalmente del estudiante, sino que gran parte de esta corresponde a factores netamente institucionales. Es importante resaltar que este modelo está basado en una de las teorías de mayor aceptación en términos de permanencia estudiantil: el modelo de abandono institucional de Tinto (1987), el cual propone que el éxito de un estudiante de una IES está mediado por expectativas, relaciones académicas y sociales dentro de la universidad y factores previos al ingreso a la institución, como orientación vocacional, estilos de aprendizaje e intereses que pueden tener incidencia en la adaptación a la vida universitaria; entre ellos, el desempeño escolar durante la educación secundaria. De esta manera, Tinto propone que existe una relación muy fuerte entre las interacciones sociales entre el educando y la institución, con la permanencia estudiantil.

A su vez, Seidman (2005) propone que una buena forma de retener al estudiante (lograr la permanencia estudiantil) es identificar alertas tempranas, en lo posible en el proceso de ingreso, para identificar las necesidades de los estudiantes y así brindar servicios y acompañamiento pertinentes para satisfacer a los usuarios de las IES. En ese orden de ideas, la permanencia estudiantil no solo es importante por el efecto que causa en los estudiantes al sentirse a gusto en la institución, sino que también, tal como lo plantea Greenberg citado por García, Núñez, Salas y Suanya (2013), implica mayores beneficios financieros para la IES; puesto que en la medida que el estudiante permanece en la universidad, el ingreso económico se mantiene estable a través del tiempo; no obstante, es necesario tener presente que para lograr esto, la IES debe hacer inversión en términos de calidad académica, tecnológica, infraestructura y servicio personalizado. Lo anterior es de suma importancia, ya que la percepción de calidad y reputación que una persona tiene sobre una IES, se convierte en uno de los primeros factores que llevan a tomar la decisión de matricularse y en el futuro a la decisión de permanecer.

Para comprender mejor el fenómeno de la permanencia estudiantil, se presentan, a continuación, dos modelos teóricos que complementan al modelo de abandono institucional de Tinto (1987) y que fueron tenidos en cuenta en el presente estudio.

Teoría de la acción razonada y permanencia estudiantil (Fishbein \& Ajzen, 1975)

Uno de los modelos pioneros en función de la permanencia estudiantil se tomó desde la perspectiva conductual, usando como base la teoría de la acción razonada (Fishbein \& Ajzen, 1975). Los autores del modelo plantean que las conductas están permeadas por las creencias y las actitudes, por lo cual la decisión de desertar o permanecer estaría relacionada con las consecuencias obtenidas en decisiones similares a lo largo de la historia de aprendizaje del sujeto. De igual forma, factores como las normas sociales que pueden desencadenar una intención 
de conducta (o, en este caso,"intención de permanencia"), generarán nuevos sistemas de creencias y actitudes. En consecuencia, en el contexto de la educación superior se asumiría la permanencia del estudiante ( $y$ las consecuencias en todas las áreas de ajuste, que ello conlleva) como un antecedente que fortalece las intenciones de conducta.

\section{Modelo de Keller brand equity basado en}

el consumidor o resonancia de marca

El modelo de Keller parte de una serie de niveles relacionados con la idea de brand equity; el primero, comprende la identidad que busca un primer acercamiento y afinidad del consumidor con la marca de tal manera que esta sea anclada en su memoria, asociándola con una necesidad específica; el siguiente, corresponde a la generación de significado de la marca en la percepción de los consumidores, para ello es necesaria la consolidación de vínculos tangibles e intangibles con la marca; en el tercer nivel se generan respuestas asociadas a sentimientos y juicios respecto a la marca; $y$ finalmente, el nivel de transformación de dichas respuestas en una relación con la marca, de forma activa y perdurable en el tiempo, por parte de los consumidores (Kotler \& Keller, 2009).

\section{El valor de marca en las IES}

En términos del marketing educativo en universidades, Kotler citado por Filip (20I2) plantea que se refiere a la planeación, implementación y control cuidadoso, para formular y diseñar programas de la institución teniendo presente las necesidades objetivo de mercado. En ese sentido, en la educación superior es importante resaltar que la formación de la imagen institucional incide en la competitividad de la compañía y el mercado laboral; dicha imagen está dada no solo por los estudiantes regulares activos, sino también en la percepción que tienen los graduados de la universidad. Por ende, tal como lo plantea Berdivayeba (20I3), deben tenerse en cuenta no solo las estadísticas, sino también identificar los estereotipos, representaciones sociales, entorno social y símbolos que existen en la comunidad frente a la IES.

Los resultados de diferentes investigaciones realizadas respecto a este tema demuestran que existen relaciones entre las representaciones simbólicas y la identidad de la marca. Lo anterior se relaciona con lo planteado por Kalafatis, Ledden, Riley y Singh (2016), quienes encontraron relaciones entre las acciones de permanencia a nivel de marketing y la construcción del valor agregado de marca. De igual manera, Yuan, Liu, Luo y Yen (2016) proponen que las acciones del marketing logran mejores procesos de identidad de marca entre los estudiantes, cuando estas se focalizan en procesos de internacionalización; además, estos autores resaltan la importancia de estudiar aspectos sociales de la permanencia e imagen institucional, en términos de representaciones sociales, estilos de consumo y de vida.

Por otro lado, Štefko, Fedorko y Bačík (20I5) proponen que el uso de la página web, e incluso las aulas virtuales (en lo que tiene que ver con diseño de la página, claridad y contenido), es un insumo importante para construir una imagen positiva y sólida en las IES; por lo cual, consideran relevante el uso del e-marketing, como herramienta fundamental para la divulgación, socialización y construcción del valor de marca. De la misma manera, Palmer, KoenigLewis y Asaad (2016) sugieren que los estudios de valor de marca deberían realizarse en diferentes instituciones y contextos, puesto que el entorno social, la cultura y la historia de cada IES, pueden arrojar resultados muy distintos en lo referente al valor de marca $y$, en ese orden de ideas, los resultados no podrían ser demasiado generalizables. Lo anterior se sustenta con lo encontrado por Rauschnabel, Krey, Babin e Ivens (20I6), quienes sugieren realizar estudios en otras IES, teniendo presente el contexto social en el que se encuentran; sin embargo, resaltan también la importancia de estudiar el tema del valor de marca como algo fuertemente relacionado con la competitividad en el mercado y la decisión de los estudiantes por permanecer dentro de la IES.

Finalmente, Girard y Trapp (2013) realizaron un estudio donde se aplicó el modelo de resonancia de marca propuesto por Keller, y encontraron que el valor de marca percibido como más importante era la calidad de la facultad a la que pertenecían los alumnos; esta percepción es considerada como más importante incluso por encima de la imagen institucional en general, la cual fue considerada como el segundo factor más importante dentro de la construcción del valor de marca. Asimismo, se encontró que los factores emocionales y de lealtad a la marca comprendieron los factores de segundo plano; no obstante, los investigadores sugieren examinar más a fondo el componente emocional dentro del modelo CBBE de Keller; también sugieren crear un instrumento de medición del valor de marca para establecer las interacciones de las múltiples dimensiones que comprenden la construcción de marca. 


\section{Metodología e instrumento}

Se hizo un muestreo no probabilístico por conveniencia, usando como tamaño de muestra el número total de estudiantes de pregrado modalidad presencial de una sede regional de una institución de educación superior privada colombiana (total de estudiantes: 3.4I5, según el Departamento de Admisión y Registro); al aplicar la fórmula correspondiente, a un nivel de confianza de $95 \%$, se estimó un total de 345 personas.

Antes de la aplicación se realizó una validación del instrumento, el cual fue diseñado especialmente para este estudio teniendo en cuenta las variables de medición de las acciones de permanencia consideradas por el Ministerio de Educación colombiano (servicio de tutorías, talleres del bienestar universitario, comunicación con docentes, inducción institucional, educación inclusiva y apoyo financiero); y las variables de resonancia de marca contenidas en el estudio de Girard y Trapp (20/3), quienes dieron el aval correspondiente para realizar los ajustes necesarios para ser aplicados en el contexto colombiano (identidad, significado, respuestas y resonancia de marca). Además, se tuvieron presentes las variables sociodemográficas y se diseñaron tres preguntas dicotómicas en las que se preguntaba la intención de permanencia (posibilidad de matrícula el próximo semestre), intención de estudiar posgrados en la IES y la intención de recomendar la IES a terceros.

Se hizo una prueba piloto, validación por jueces y validación estadística, obteniendo un índice alfa de Cronbach de 0,89 lo cual indicó consistencia interna del instrumento. El estudio partió de un diseño cuantitativo de corte descriptivo correlacional, cuyo objetivo fue encontrar la relación existente entre las variables de valor de marca según el modelo de Keller, brand equity. Basado en el consumidor y la intención de permanencia estudiantil.

Para el análisis estadístico se utilizó el software SPSS, con el que se calcularon: coeficiente alfa de Cronbach, estadísticos descriptivos, pruebas de normalidad, frecuencias, Chi cuadrado y coeficiente de correlación de Spearman; participaron en el estudio 453 estudiantes. Se tuvo en cuenta la totalidad de respuestas obtenidas en un intervalo de 30 días.

\section{Consideraciones éticas}

De acuerdo con los principios establecidos en la Ley 1090 de 2006, que regula el ejercicio profesional de la psicología en Colombia, el presente estudio se rigió por los siguientes criterios:

Específicamente, el artículo 20 de la Ley 1090 de 2006 en la que se indica que cuando se halle ante intereses personales o institucionales contrapuestos, el profesional realizará su actividad en términos de máxima imparcialidad. La prestación de servicios en una institución no exime de la consideración, respeto y atención a las personas que pueden entrar en conflicto con la institución misma y de las cuales en aquellas ocasiones en que legítimamente proceda, habrá de hacerse valedor ante las autoridades institucionales

De acuerdo con lo anterior, se solicitó el debido permiso para el acceso a la población modalidad presencial de la institución, para realizar el presente estudio; dicho documento fue firmado por el director de la institución elegida, donde manifestaba estar de acuerdo con la aplicación del estudio e incentivo económico por participación que se haría mediante sorteo para los estudiantes participantes. Además, se tuvieron en cuenta los siguientes artículos de la Ley 1090 de 2006 para la realización de la investigación:

Artículo 50. Los profesionales de la psicología al planear o llevar a cabo investigaciones científicas, deberán basarse en principios éticos de respeto y dignidad, lo mismo que salvaguardar el bienestar y los derechos de los participantes.

Artículo $5 \mathrm{Ic}$ ). Que se garantice que al terminar la investigación se les va a brindar a los participantes la información correcta sobre las variables utilizadas y los objetivos de la investigación. [Bajo este artículo se le socializó a cada participante (una vez finalizaba el instrumento) el correo electrónico del investigador para que pudiese solicitar los resultados generales del estudio mediante correo electrónico].

Artículo 56. Todo profesional de la psicología tiene derecho a la propiedad intelectual sobre los trabajos que elabore en forma individual o colectiva, de acuerdo con los derechos de autor establecidos en Colombia. Estos trabajos podrán ser divulgados o publicados con la debida autorización de los autores.

\section{Análisis y resultados}

\section{Prueba Chi cuadrado}

Para llevar a cabo las pruebas de hipótesis se tomaron los ítems correspondientes a cada una de las fases del modelo 
brand equity basada en el consumidor (Keller): identidad, significado y respuestas. Cada uno de los ítems se cruzaron con las tres variables dicotómicas del estudio: intención de permanencia, intención de estudiar posgrado y recomendar la IES a terceros; esta prueba estadística se aplicó con el fin de identificar los elementos del modelo de Keller que se relacionan con la variables. Los resultados con valores estadísticamente significativos se muestran a continuación.

Tabla I. Tabla cruzada de la variable dimensión identidad de la marca con recomendar la IES a externos

\begin{tabular}{|c|c|c|c|c|c|}
\hline & & & \multicolumn{2}{|c|}{$\begin{array}{l}\text { ¿Recomendaría a } \\
\text { personas externas } \\
\text { estudiar en la } \\
\text { institución en la } \\
\text { que realizo/realicé } \\
\text { mis estudios? }\end{array}$} & \multirow[t]{2}{*}{ Total } \\
\hline & & & No & Sí & \\
\hline \multirow{3}{*}{$\begin{array}{l}\text { Que la } \\
\text { universidad } \\
\text { tenga } \\
\text { prestigio } \\
\text { a nivel } \\
\text { nacional }\end{array}$} & $\begin{array}{c}\text { Poco } \\
\text { importante }\end{array}$ & $\begin{array}{c}\text { Recuento } \\
\text { Residuo } \\
\text { corregido }\end{array}$ & $\begin{array}{c}0 \\
-1,2\end{array}$ & $\begin{array}{l}21 \\
1,2\end{array}$ & 21 \\
\hline & Importante & $\begin{array}{c}\text { Recuento } \\
\text { Residuo } \\
\text { corregido }\end{array}$ & $-2,2$ & 127 & 130 \\
\hline & $\begin{array}{c}\text { Muy } \\
\text { importante }\end{array}$ & $\begin{array}{l}\text { Recuento } \\
\text { Residuo } \\
\text { corregido }\end{array}$ & 25 & 277 & 302 \\
\hline \multicolumn{2}{|c|}{ Total } & Recuento & 28 & 425 & 453 \\
\hline
\end{tabular}

El valor de la significancia asintótica fue de 0,030 para este caso. Fuente: elaboración propia.

La tabla I presenta las diferencias estadísticas en términos de las frecuencias de las variables de interés $(p=0,05)$; es decir, se identifica que hay más personas que recomendarían a terceros la universidad en la que estudian, considerando importante el prestigio que tiene la universidad a nivel nacional.

Para la dimensión significado, se obtuvieron los resultados de la tabla 2, la cual cruza las variables intención de permanencia y la existencia de programas de internacionalización. Puede apreciarse que hay una mayor cantidad de personas que consideran muy importante la existencia de oportunidades de internacionalización con la intención de renovar la matrícula el próximo semestre; lo que puede indicar que el comportamiento de las variables no corresponde a aspectos aleatorios. De esta manera, se identifica que probablemente este es el elemento de la marca que más aportaría a la permanencia estudiantil y (como se vio anteriormente) a la resonancia de marca, confirmando así la hipótesis de este estudio.
Tabla 2. Tabla cruzada de la variable oportunidades de internacionalización con intención de permanencia

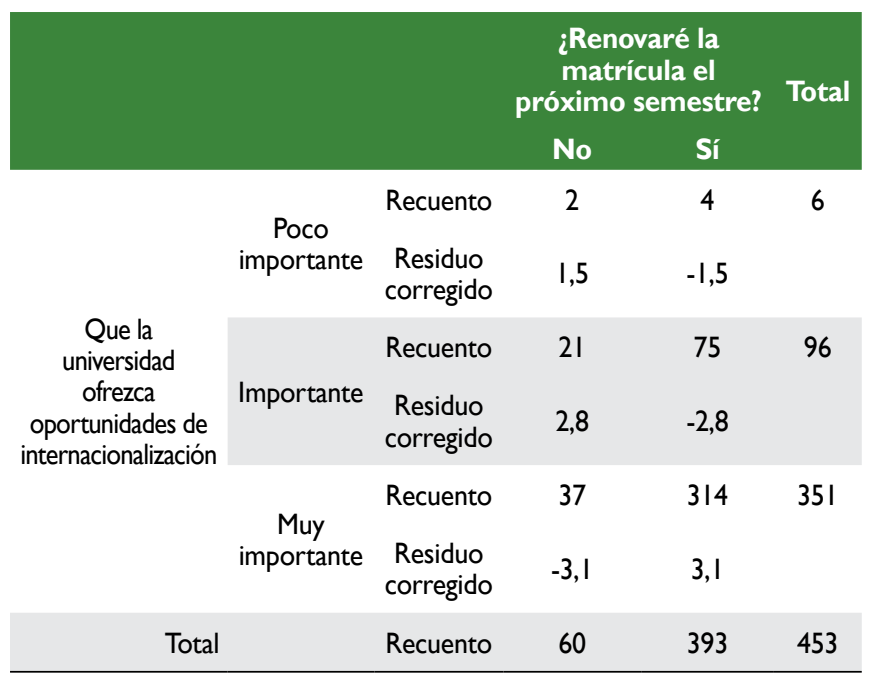

El valor de la significancia asintótica fue de 0,005 para este caso.

Fuente: elaboración propia.

La tabla 2 muestra que únicamente los procesos de internacionalización se relacionan con la intención de permanencia dentro de la medición realizada; aunque se sometieron los otros ítems correspondientes a todas las fases del modelo de Keller, son solo los procesos de internacionalización (medido en la dimensión significados de la marca) parecen relacionarse de forma significativa con la intención de permanecer en la institución, por sobre las otras variables medidas con este instrumento.

\section{Coeficiente de correlación de Spearman}

Para estudiar la asociación entre los factores I y 2 de la escala, estos se correlacionaron utilizando una correlación de Spearman y con este coeficiente se obtuvo una correlación de 0,997 (véase tabla 3), lo que indica que hay una fuerte asociación entre las variables acciones de permanencia y valor de marca.

La correlación que se observa en la tabla 3 , indica que a medida que la percepción de las acciones de permanencia sea positiva, el valor de marca va adquiriendo mayor importancia.

\section{Discusión}

Los resultados permiten establecer que existe una relación significativa $(r=0,99)$ entre las acciones de permanencia estudiantil (intervención psicosocial individual y grupal, cursos de cultura y deporte, subsidios económicos, tutorías 
Tabla 3. Correlación de Spearman entre acciones de permanencia y valor de marca

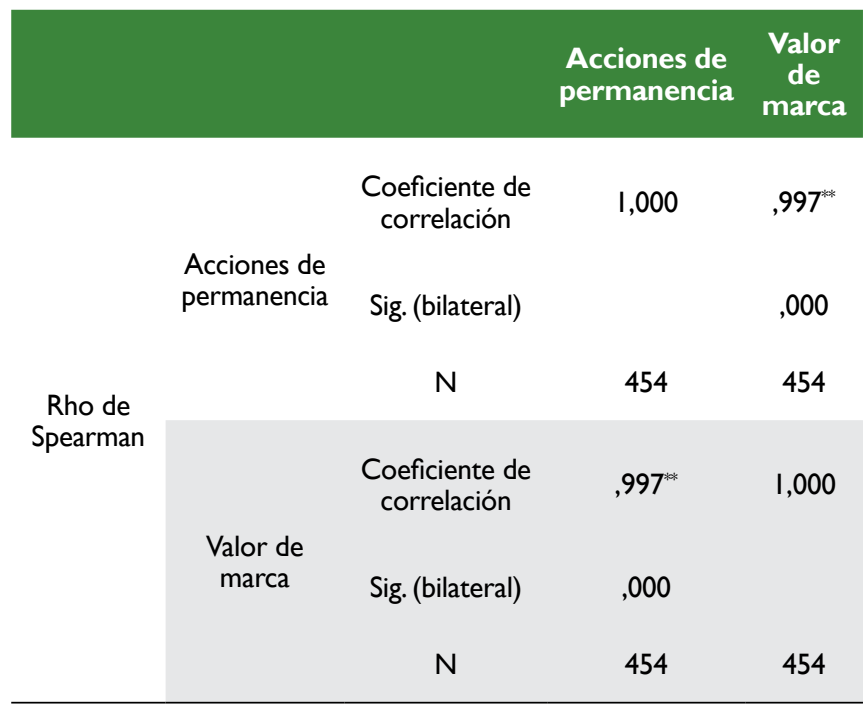

Los valores $p$ inferiores a 0,05 indican que el comportamiento de los grupos, respecto a los dos factores, no ocurren de forma aleatoria; por tal motivo, se podría rechazar la hipótesis nula.

Fuente: elaboración propia

académicas entre pares y con docentes, procesos de educación inclusiva y demás actividades propias del bienestar universitario) con la importancia que tiene la marca para el estudiante, lo cual implica decir que entre mejor percepción se tengan de las acciones de permanencia, el valor de marca adquiere mayor importancia para los estudiantes. Este resultado coincide perfectamente por lo planteado en Kalafatis et al. (2016), quienes manifiestan que las acciones de permanencia estudiantil fortalecen la construcción del valor de la marca en instituciones de educación superior; asimismo, se relaciona con lo encontrado en el estudio Dennis, Papagiannidis, Alamanos y Bourlakis (2016), donde argumentan que la retención estudiantil está fuertemente relacionada con variables de la marca.

De igual forma, se identificó que entre más importancia adquieren los procesos de internacionalización para el estudiante, tanto el vínculo emocional con la universidad como la intención de permanencia aumenta; estos resultados se relacionan con lo encontrado por Yuan et al. (2016), quienes afirmaron en su estudio que la identidad de la marca se fortalece en la medida que las estrategias de marketing se focalicen a los procesos de internacionalización de la universidad. Para el caso de este estudio, se añade la fase de significado como aspecto que también le aporta a la resonancia de marca.

Los anteriores resultados muestran concordancia con los modelos de la acción razonada (Fishbein \& Ajzen, 1975) y el modelo del abandono institucional (Tinto, 1987); pues es justamente gracias a las interacciones que se dan dentro de la institución que los estudiantes pueden retroalimentar positiva o negativamente sus expectativas $y$, por ende, decidir si permanecen o desertan de la institución. Los elementos del valor de marca, según el modelo de Keller (1993), que adquieren importancia en esta correlación son la identidad, significado y las interacciones (respuestas) de los estudiantes con la marca y se relacionan con las acciones académicas y del bienestar universitario en función de la permanencia estudiantil. Por esta razón, los resultados aportan un elemento interesante que puede ser transversal en el modelo de abandono institucional, permitiendo a los investigadores del presente estudio proponer un nuevo modelo de permanencia estudiantil (véase figura I), donde gracias a las dinámicas de las interacciones en la integración académica y social que tenga el estudiante, este va construyendo el valor de la marca de la IES, lo cual resultaría pertinente para fortalecer los procesos de identidad y significado institucional (o sentido de pertenencia) y en el futuro el vínculo emocional con la marca.

Este nuevo modelo combina el marketing educativo, la perspectiva conductual de la permanencia estudiantil y rescata los elementos más valiosos del modelo organizacional del abandono, generando una alternativa integral para el fortalecimiento de las relaciones que puedan crearse entre el estudiante y cada instancia de la universidad en función del cumplimiento de sus expectativas y objetivos; este modelo supone que la experiencia del sujeto dentro del contexto universitario es fundamental para la marca global y la continuidad de sus estudios.

Para comprender mejor esta nueva propuesta sugerimos tener en cuenta los factores que se consideran originalmente en el modelo de abandono institucional, tales como historia de aprendizaje del estudiante, aspectos personales y orientación vocacional. De acuerdo con estos, el sujeto busca la IES en la que desea estudiar teniendo en cuenta el reconocimiento o posicionamiento de la IES en el contexto nacional (identidad de la marca), las asociaciones que se tenga de la institución (por ejemplo, enfoque de la universidad, discursos asociados, etc. — significado de la marca-) y las respuestas hacia la marca (es decir, las posibles interacciones que haya tenido el individuo $o$ un allegado con una o algunas instancias de la IES).

Teniendo presente lo anterior, el aspirante toma la decisión de ingresar a la institución y una vez dentro, el ahora 


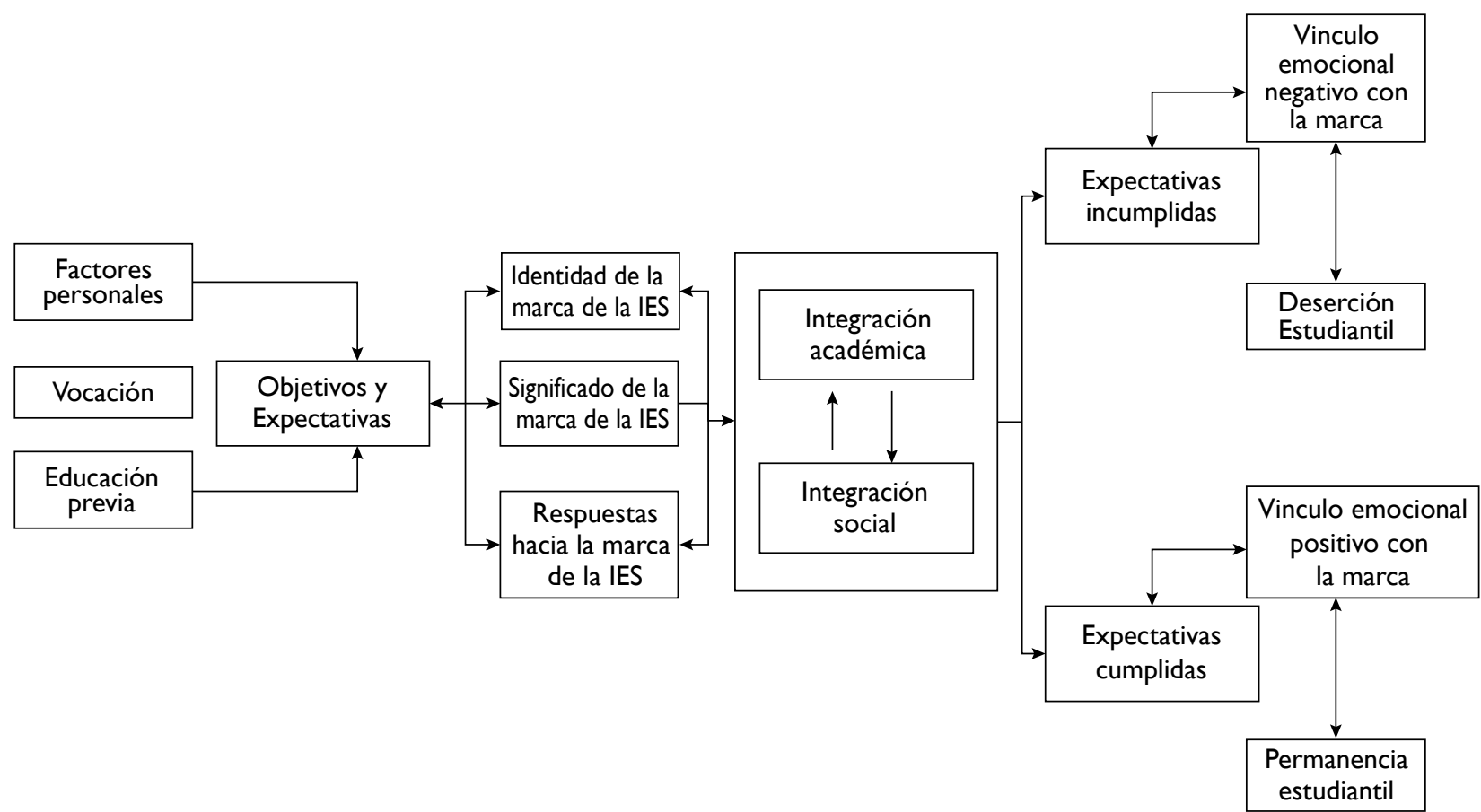

Figura I. Modelo de permanencia estudiantil y resonancia de marca en instituciones de educación superior. Fuente: elaboración propia con base en los resultados de esta investigación y los modelos teóricos de base.

estudiante se ve inmerso en la integración académica y social que propone Tinto (1987), en donde las interacciones con docentes y compañeros, así como el rendimiento académico y las actividades extracurriculares (generalmente asociadas al bienestar universitario), tienen un papel importante en el cumplimiento o no de las expectativas previas retroalimentando las tres fases del modelo brand equity basado en el consumidor, ya mencionadas, fortaleciéndolas o debilitándolas.

Así, de acuerdo con las consecuencias recibidas en la dinámica de la integración académica y social, el estudiante podría tomar la decisión de permanecer o desertar, generando, a su vez, un vínculo emocional con la marca (resonancia de marca). Si la expectativa del estudiante se cumple (es reforzada positivamente), puede generar un vínculo emocional positivo con la marca, que da lugar a la permanencia estudiantil $y$, por ende, a la probabilidad de recomendar la universidad a otros o continuar estudiando en la IES, posterior al grado. En el caso contrario, cuando las expectativas no son cumplidas y se obtiene una integración académica y social negativa (castigo), posiblemente el vínculo emocional con la marca será negativo y aumentará la probabilidad de deserción e implicará, por consiguiente, menor probabilidad de recomendar la IES a personas externas en el futuro.
Por otro lado, es importante tener en cuenta las siguientes consideraciones para aplicación y futuras investigaciones:

En primer lugar, la correlación encontrada entre el valor de marca y las acciones de permanencia, puede aportar un elemento importante para las estrategias que se diseñan desde las IES para el fomento de la permanencia estudiantil, ya que al incluir elementos del valor de marca sugiere que este tipo de acciones (generalmente ejecutadas desde el área de bienestar universitario) tengan un complemento con el marketing educativo para consolidar procesos de vínculos emocionales con la marca y cumplimiento de expectativas que propicien la permanencia estudiantil. De igual forma, al encontrar que la percepción positiva de las acciones de permanencia aumenta el valor de marca percibido, se recomienda que los departamentos que realizan las acciones de permanencia (bienestar universitario, departamento de egresados, departamento de internacionalización, etc.) utilicen su nombre de área únicamente con fines de los procesos internos de la IES; en otras palabras, se sugiere el fortalecimiento de la marca global de la IES y no la del departamento que brinda el servicio.

A la luz de los resultados, estas acciones lograrían que los significados y respuestas hacia la marca se fortalezcan y se vean reflejados en el vínculo emocional que se 
pretende crear dentro del modelo de resonancia de marca propuesto. Sin embargo, debe tenerse especial cuidado, ya que estos resultados se extraen de la muestra de una sola institución educativa. Si bien los resultados muestran coherencia y relación con investigaciones previas, se sugiere para futuras investigaciones tomar muestras en más de una institución de educación superior, con el fin de establecer si estos resultados pueden generalizarse a otras instituciones o constituyen una particularidad de la institución en la que se realizó este estudio. Relacionado con lo anterior, la muestra escogida para el presente estudio corresponde a una universidad ubicada en un contexto regional, es posible que las características propias de la región y el perfil psicodemográfico del contexto afecten las respuestas e intenciones de permanencia, lo que se manifestaría en resultados distintos en universidades de ciudades capitales; esta observación surge de lo estipulado por Palmer et al. (2016), quienes sugieren que el entorno social en el que se encuentra la universidad, así como factores culturales e históricos, podría alterar la percepción y el valor de la marca en instituciones. De igual manera, se sugiere que en estudios futuros se tengan en cuenta otros actores de la institución que pudiesen dar un panorama más amplio de la importancia del valor de la marca en la permanencia estudiantil (por ejemplo: padres de familia, estudiantes desertores, ausentes y graduados) usando un muestreo aleatorio simple.

En el presente trabajo se utilizó un muestreo no probabilístico por conveniencia en una sola institución educativa; sin embargo, los resultados obtenidos están lejos de convertirse en un estudio de mercados de la IES participante. Por el contrario, la investigación aporta aspectos generales encontrados en otras universidades alrededor del mundo (identidad de la marca, procesos de internacionalización, integración académica y social, significados y respuestas de la marca asociados al valor de marca, la intención de permanencia y la probabilidad de recomendar la IES a personas externas).A modo de conclusión general, el hecho de que los resultados hayan coincidido con los resultados de investigaciones anteriores en contextos diferentes, puede dar un sustento sólido del aporte conceptual que el presente estudio entrega a las estrategias de permanencia estudiantil en las instituciones de educación superior y puede servir de fundamento teórico para abordar la retención desde una perspectiva institucional que pretenda retroalimentar el servicio de la educación constantemente, para así satisfacer las necesidades y expectativas específicas de sus usuarios.

\section{Referencias}

Banco Mundial. (17 de mayo de 2017). La educación superior se expande en América Latina y el Caribe, pero aún no desarrolla todo su potencial. Recuperado de http://www.bancomundial. org/es/news/press-release/2017/05/17/higher-educationexpanding-in-latin-america-and-the-caribbean-but-fallingshort-of-potential.

Berger, J. B., Blanco-Ramírez, G., \& Lyon, S. (2012). Past to present: A historical look at retention (pp. 7-34). In A. Seidman (Ed.), College student retention: Formula for student success. Recuperado de https://www.academia. edu/604908I/Past_to_present_A_historical_look_at_ retention?auto=download.

Berdibayeva, S. K., Maigeldiyeva, S. M., Tazhbayeva, S. G., Abirova, M.A., \& Atakhanova, S. K. (2014). The Image of Pedagogical Higher Education Institution as Psycho-pedagogical Problem. Procedia - Social and Behavioral Sciences, II6, 294I-2945. http://doi.org/10.1016/j.sbspro.2014.01.684

Denegri, M., Cabezas, D., Novoa, M., Peralta, J., \& Estrada, C. (2013). Personalidad de marca en carreras de psicología de zonas territoriales extremas: Arica y Punta Arenas. Magallania, 4 I (85-I00).

Dennis, Papagiannidis, Alamanos, \& Bourlakis. (2016). The role of brand attachment strength in higher education. Journal of Business Research,(I), 65, doi:10.1016/j.jbusres.2016.01.020.

Filip,A. (2012). Marketing theory applicability in higher education. Procedia - Social and Behavioral Sciences, 46, 912-916. doi:10.1016/j.sbspro.2012.05.223.

Fishbein, M., \& Ajzen, I. (1975). Belief, attitude, intention and behavior:An introduction to theory and research. Recuperado de https://www.researchgate.net/publication/233897090 Belief_attitude_intention_and_behaviour_An_ introduction_to_theory_and_research.

Fonseca, G., \& García, F. (2016). Permanencia y abandono de estudios en estudiantes universitarios: un análisis desde la teoría organizacional. Revista de la Educación Superior. http://doi.org//0.1016/j.resu.2016.06.004.

García, C., Núñez, R., Salas, N., \& Suanya, O. (20l3). El cliente social: retos de la atención al cliente en el universo de las redes sociales.

Girard, T., \& Trapp, P. (2013). University brand equity: An empirical investigation of its dimensions. Recuperado de https://www. academia.edu/22996 107/University_brand_equity_an_ empirical_investigation_of_its_dimensions?auto=download.

Kalafatis, S. P., Ledden, L., Riley, D., \& Singh, J. (20I6). The added value of brand alliances in higher education.Journal of Business Research. http://doi.org/I0.1016/j.jbusres.2016.01.028. 
Keller, K. (1993). Conceptualizing, measuring, and managing customer-based brand equity. Journal of Marketing, 57(I), I-22. doi: I0.2307// 252054.

Kotler, P., \& Keller, K. (2009) Dirección de marketing. México: Pearson Educación.

Ley 1090 de 2006."Por la cual se reglamenta el ejercicio de la profesión de Psicología, se dicta el Código Deontológico y Bioético y otras disposiciones". Recuperado de http://www.secretariasenado.gov.co/senado/basedoc/ ley_1090_2006.html

Ministerio de Educación Nacional. (2009). Deserción estudiantil en la educación superior colombiana. Metodología de seguimiento, diagnóstico y elementos para su prevención. Bogotá: Imprenta Nacional de Colombia.

Ministerio de Educación Nacional. (20I5). Estrategias para la permanencia en educación superior: experiencias significativas. Bogotá: Ed. Qualificar.

Palmer, A., Koenig-Lewis, N., \& Asaad, Y. (2016). Brand identification in higher education: A conditional process analysis.Journal of Business Research.http://doi.org/10.1016/j. jbusres.2016.01.018

Rauschnabel, P. A., Krey, N., Babin, B. J., \& Ivens, B. S. (20I6). Brand management in higher education: The university brand personality scale. Journal of Business Research. http://doi.org/10.1016/j.jbusres.2016.01.023.
Seidman, A. (2005). Minority student retention: Resources for practitioners. New Directions for Institutional Research, 125, 7-24. Recuperado de http://www.imsd.emory.edu/ documents/documents-pdfs/minority-retention-seidman.pdf.

Štefko, R., Fedorko, R., \& Bačík, R. (20I5).The role of e-marketing tools in constructing the image of a higher education institution. Procedia - Social and Behavioral Sciences, I75, 43 I-438. http://doi.org/10.1016/j.sbspro.2015.01.1220

Tinto, V. (1987). Leaving college. Rethinking the causes and Cures of student attrition. Chicago:The University of Chicago press.

Torres, L. (2012). Retención estudiantil en la educación superior. Recuperado de http://www.javeriana.edu.co/documents/I 5838/273636/Retenci\%C3\%B3nEstudiantil20I 2. pdf/I 24fdba5-23 I 8-432a-8e9f- I 26a250 I c229.

Yuan, R., Liu, M. J., Luo, J., \& Yen, D.A. (2016). Reciprocal transfer of brand identity and image associations arising from higher education brand extensions. Journal of Business Research, 69(8), 3069-3076. http://doi.org/10.1016/j. jbusres.2016.01.022 